\title{
Cryptdin-2 predicts intestinal injury during heatstroke in mice
}

\author{
JINGJING JI ${ }^{1,2^{*}}$, ZHENGTAO GU $^{3 *}, \mathrm{HUI} \mathrm{LI}^{3}$, LEI SU ${ }^{1,3}$ and ZHIFENG LIU ${ }^{1,3}$ \\ ${ }^{1}$ Department of Critical Care Medicine, General Hospital of Guangzhou Military Command; \\ ${ }^{2}$ Graduate School, Guangzhou Medical University; ${ }^{3}$ Key Laboratory of Hot Zone Trauma Care and Tissue Repair of PLA, \\ General Hospital of Guangzhou Military Command, Guangzhou, Guangdong 510010, P.R. China
}

Received March 30, 2017; Accepted October 27, 2017

DOI: $10.3892 /$ ijmm.2017.3229

\begin{abstract}
Intestinal injury-induced bacterial translocation and endotoxemia are important in the pathophysiological process of heatstroke. However, the underlying mechanism remains to be fully elucidated. Previous studies using 2D-gel electrophoresis found that defensin-related cryptdin-2 (Cry-2), an intestinal $\alpha$-defensin, is upregulated in intestinal tissues during heatstroke in mice, and that treatment with ulinastatin, a multivalent enzyme inhibitor, reduced heat-induced acute lung injury. To investigate the association between Cry-2 and heat stress (HS)-induced intestinal injury and the probable protective role of ulinastatin, the present study examined the intestinal expression of Cry-2 via histopathologic analysis and reverse transcription-quantitative polymerase chain reaction analysis in mice with heatstroke. The heat-stressed mice were exposed to different core temperatures and cooling treatments, and intestinal pathological changes and Chiu scores were determined. Chemical markers of intestinal injury, serum and intestinal concentrations of diamine oxidase (DAO) and D-lactic acid (D-Lac), and serum and intestinal concentrations of Cry-2 were also determined. Correlations were analyzed using Spearman's correlation analysis. It was found that HS upregulated the expression of Cry-2, and the serum and intestinal concentrations of Cry- 2 were correlated with the severity of HS-induced intestinal damage, indicated by pathology scores and concentrations of DAO and D-lac. Ulinastatin protected the intestines from HS-induced injury and downregulated the expression of Cry-2, which was also correlated with the extent of intestinal injury. Therefore, ulinastatin administration may be beneficial for patients with heatstroke, and Cry- 2 may be a novel predictor of HS-induced intestinal injury.
\end{abstract}

Correspondence to: Professor Zhifeng Liu, Department of Critical Care Medicine, General Hospital of Guangzhou Military Command, 11 Liuhua Road , Guangzhou, Guangdong 510010, P.R. China E-mail: zhifengliu7797@163.com

${ }^{*}$ Contributed equally

Key words: heatstroke, intestinal injury, defensin, cryptdin-2, ulinastatin

\section{Introduction}

Heatstroke is a life-threatening illness, which is characterized by a core temperature (Tc) $>40^{\circ} \mathrm{C}$ and central nervous system dysfunction, and is associated with increased morbidity and high mortality rates $(1,2)$. However, the potential mechanism underlying the high mortality rates in heatstroke remains to be fully elucidated, and there is a lack of targeted and effective treatment. As the gut contains a large pool of bacteria and endotoxins, intestinal injury, and the increased permeability-induced bacterial translocation and endotoxemia have been implicated in the pathophysiological process of heatstroke $(3,4)$. However, the molecular changes underlying small-intestine lesions during heat stress (HS) remain to be fully characterized. Our previous study used 2D-gel electrophoresis technology to identify 14 differentially expressed proteins in the small intestines of mice subjected to HS. These 14 proteins may be involved in metabolism, chaperone functions, the cytoskeleton, defense, signal transduction, and DNA repair and recombination. Intestinal defensin-related cryptdin-2 (Cry-2), a member of the $\alpha$-defensin family of peptides, is upregulated in the small intestinal tissue of heat-stressed mice (5).

Increasing evidence suggests that paneth cells, which are found in the small intestines of mammals, are involved in the mucosal barrier function (6) by secreting apical granules containing antimicrobial peptides, including $\alpha$-defensins, which are termed cryptdins in mice (7). Cry-2 has potent antimicrobial activity against certain microbes, including Escherichia coli and Salmonella typhimurium $(8,9)$. Previous results suggest that luminal bacteria can increase the expression of Cry-2, particularly in the ileum (10). In addition to their effects on microbes, cryptdins also assist in regulating the innate inflammatory response and cell death $(11,12)$. However, the role of Cry-2 in HS-induced mouse intestinal injury remains to be fully elucidated. In order to confirm the possible association between intestinal Cry-2 and the severity of intestinal injury, the present study measured intestinal tissue injuries and levels of Cry-2 in the ileal tissues of heat-stressed mice, and analyzed the correlations between them.

Two biomarkers are traditionally used to assess enterocytic damage and dysfunction, namely diamine oxidase (DAO) and D-lactic acid (D-Lac). DAO is an enzyme of the catabolic pathway and normally is present in intestinal mucosa and villi. During ischemia, hypoxia, or sepsis, DAO can be released into the circulation, and its serum concentration is positively 
correlated with the integrity of the intestinal mucosa $(13,14)$. D-Lac is produced by several types of bacteria, and its increased concentration in serum indicates that either the permeability of the intestinal wall is increased or that elevated bacterial reproduction is occurring $(15,16)$. In order to evaluate the severity of HS-induced intestinal injury, the present study performed intestinal histopathological analyses, measured pathological scores, and measured serum and intestinal concentrations of DAO and D-Lac.

Ulinastatin, a multivalent enzyme inhibitor, was first identified and purified from human urine. Previous studies have shown that ulinastatin can inhibit the inflammatory response by decreasing associated mediators, including highmobility group box 1 and tumor necrosis factor- $\alpha(17,18)$. Pharmacological studies have shown that this anti-inflammatory activity is the result of suppressing the infiltration of neutrophils, and the production and secretion of elastase and chemical mediators by macrophages and neutrophils $(19,20)$. In addition, ulinastatin can attenuate oxidative damage (21). Clinically, ulinastatin has been used for the treatment of patients with pancreatitis and multiple organ dysfunction syndrome (MODS) $(22,23)$. Our previous study demonstrated that ulinastatin reduced pulmonary edema and inflammatory exudation in acute lung injury caused by heatstroke (24), possibly on the basis of its anti-inflammatory effects. Therefore, the present study aimed to investigate whether ulinastatin can reduce intestinal damage in heatstroke; interventions with ulinastatin combined with cooling treatments were also investigated to further elucidate the association between Cry-2 and intestinal damage.

\section{Materials and methods}

Animals. A total of 60 the pathogen-free male BALB/c mice, 6-8-week-old and weighing 18-22 g, were purchased from the Experimental Animal Center of Southern Medical University (Guangzhou, China). The mice were housed in cages with a temperature of $23^{\circ} \mathrm{C}$ and humidity of $55 \%$ (12-h light/dark cycle), and they were given free access to standard food and water. All animal procedures were approved by the Animal Care and Use Committee of Southern Medical University, and the study was performed according to the Guidelines for Animal Care of Southern Medical University.

Heat-stress protocol and cooling treatment. Heat-stress in the mice was established according to our previously reported method (25). Briefly, the mice were fasted for $12 \mathrm{~h}$ prior to the experiment without limitation on the ingestion of water. The animals in the HS group were placed in a prewarmed incubator, which was maintained at $35.5 \pm 0.5^{\circ} \mathrm{C}$ with a relative humidity of $60 \pm 5 \%$, with the absence of food and water. The rectal Tc was monitored every $30 \mathrm{~min}$ with a rectal thermometer. When the Tc reached 40 or $42^{\circ} \mathrm{C}$, respectively, the mice in these two groups were sacrificed. The mice in another two groups, $40^{\circ} \mathrm{C}\left(40^{\circ} \mathrm{C} / 6 \mathrm{~h}\right)$ and $42^{\circ} \mathrm{C}\left(42^{\circ} \mathrm{C} / 6 \mathrm{~h}\right)$, were allowed to cool at an ambient temperature of $25 \pm 0.5^{\circ} \mathrm{C}$ and a humidity of $35 \pm 5 \%$ for $6 \mathrm{~h}$ subsequent to their Tc reaching 40 or $42^{\circ} \mathrm{C}$, prior to sacrifice. The animals in the sham control group were heated to a temperature of $25 \pm 0.5^{\circ} \mathrm{C}$ and a humidity of $35 \pm 5 \%$ for a time comparable to that of the HS groups and the cooling treatment groups $\left(37^{\circ} \mathrm{C}\right.$ and $37^{\circ} \mathrm{C} / 6 \mathrm{~h}$, respectively). All groups contained six animals.

Identification of cryptdin-2 using $2 D$ gel electrophoresis, matrix-assisted laser desorption/ionization-time-of-flight mass spectrometry (MALDI-TOF MS) and database analysis. The identification of cryptdin-2 by 2D gel electrophoresis MALDI-TOF MS and database analysis were performed according to our previously reported method (Applied Biosystems; Thermo Fisher Scientific, Inc., Waltham, MA, USA) (5). Protein spots were analyzed using ImageMaster 2D Elite (GE Healthcare Life Sciences, Pittsburgh, PA, USA) and a comparative sequence search was performed in the Mascot database (http://www.matrixscience.com). Enlarged images from the 2D gels of spot H1 and its MALDI-TOF MS identification were obtained.

Histopathological analysis of intestines and intestinal crypts. The mice were anesthetized by intraperitoneal injection of chloral hydrate. Ileal samples were separated and fixed in $4 \%$ paraformaldehyde, followed by embedding in paraffin blocks. Serial sections with dimensions of $5 \mathrm{~mm}$ were stained with hematoxylin and eosin for microscopic evaluation at a magnification of x200 under a microscope (90I; Nikon, Tokyo, Japan). Morphologic changes were assessed and graded on a scale of 0-5 using the intestinal injury score developed by Chiu et al (26). Based on mucosal changes, the grades were assessed between 0 and 5 as follows: Grade 0, normal mucosal villi; grade 1, subepithelial space can be seen at the apex of the villus; grade 2, sections with extension of the subepithelial space and moderate lifting of the epithelial layer from the lamina propria; grade 3, massive epithelial lifting down the sides of villi; grade 4, sections with denuded villi and dilated capillaries; and grade 5, sections with digestion and disintegration of lamina propria. Morphological changes of the intestinal crypts and the evaluation of defensin (red dye particles) secreted by paneth cells were also detected (magnification, $\mathrm{x} 400$ ).

Reverse transcription-quantitative polymerase chain reaction (RT-qPCR) identification of Cry-2. The mice were subjected to $\mathrm{HS}$ and were sacrificed when their Tc reached 40 or $42^{\circ} \mathrm{C}$, as described above. Ileal samples were separated, and RT-qPCR analysis was performed to examine the mRNA levels of Cry-2. Total RNA was isolated using a total RNA purification kit (Promega Corp., Madison, WI, USA) and reverse transcribed using ReverTra Ace (Toyobo Co., Ltd., Osaka, Japan). PCR amplification (50 ng cDNA, $40 \mathrm{nM}$ primers, cycling conditions: $95^{\circ} \mathrm{C} 20 \mathrm{sec}$ and followed by $95^{\circ} \mathrm{C} 1 \mathrm{~min}, 60^{\circ} \mathrm{C} 20 \mathrm{sec}$ for 40 cycles) was performed and analysis according to the protocol of fluorescence quantitative kit (SYBR Premix Ex Taq; Takara Holdings Inc., Kyoto, Japan) and Agilent StrataGene Mx3005P qPCR system (Agilent Technologies Inc., Santa Clara, CA, USA) on the resulting cDNA samples using specific primers for Cry-2 (Cry2 forward, 5'-TCTCCTTTGGAGACCCAGAA-3' and reverse, 5'-CAGGCGTTCTCTTCTTTTGC-3') and glyceraldehyde 3-phosphate dehydrogenase (GAPDH forward, 5'-ATTGTCAGCAATGCATCCTG-3' and reverse, 5'-ATGGAC TGTGGTCATGAGCC-3'). The expression results of Cry-2 RT-qPCR were calculated by the $2^{-\Delta \Delta \mathrm{Cq}}$ method reported by 
A
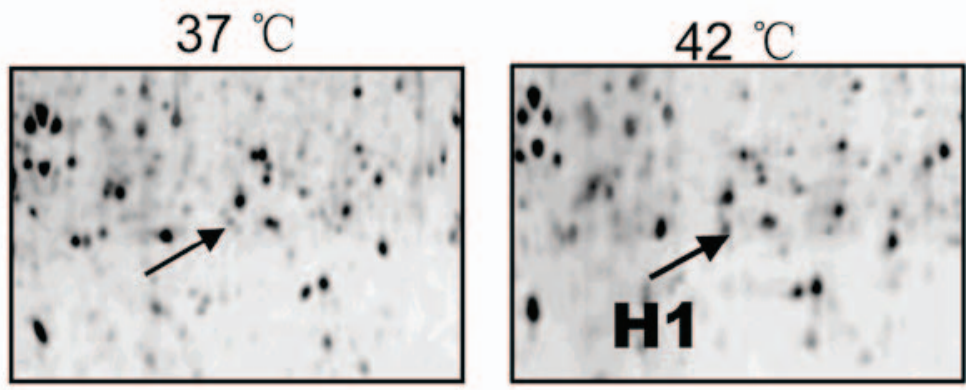

B

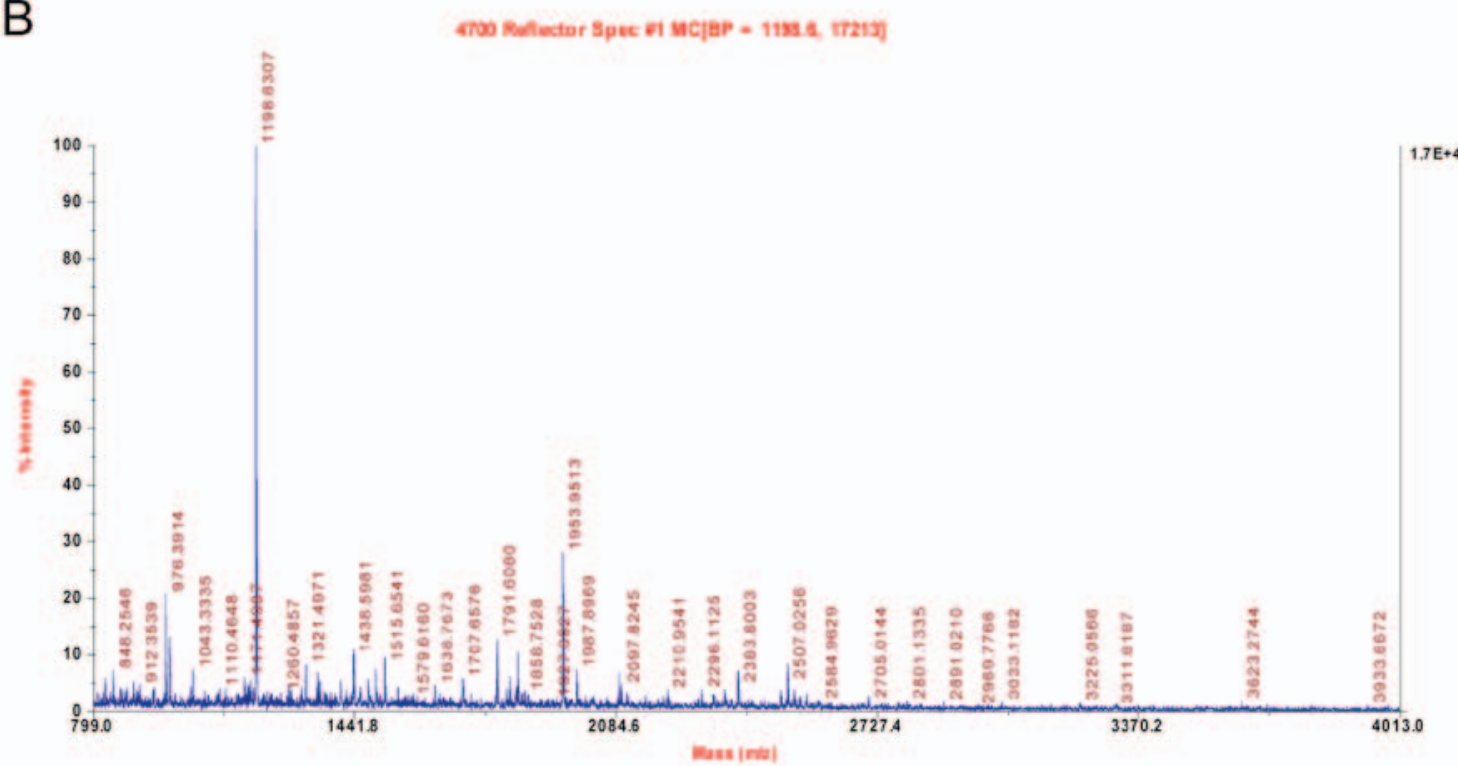

Figure 1. (A) Images from the 2D electrophoresis gels of spot H1 and its (B) matrix-assisted laser desorption/ionization-time-of-flight mass spectrometry identification. The arrows indicate the protein spot H1, subsequently identified as cryptdin-2, from ileal tissues of the control and heat-stressed mice. Spot H1 was markedly upregulated in the heat-stressed mice, compared with that in the control mice.

Livak and Schmittgen (27) and shown as the ratio of Cry-2 over GAPDH, analyzed.

Ulinastatin treatment. The mice received ulinastatin by intraperitoneal injection at a dose of $10 \mathrm{WU} / \mathrm{kg}$ immediately following the Tc reaching 40 or $42^{\circ} \mathrm{C}$. The mice in the shamheated group received the same treatment at the same time. Tissue samples were extracted $6 \mathrm{~h}$ following ulinastatin treatment.

Blood collection and determination of serum levels of DAO, $D$-Lac and Cry-2. The mice were anesthetized with chloral hydrate by intraperitonal injection, following which blood samples were obtained from the eyes, placed into $1.5-\mathrm{ml}$ heparinized microcentrifuge tubes, and placed immediately on ice for $1 \mathrm{~h}$. The serum was separated by centrifugation at room temperature for $5 \mathrm{~min}$ at $1,200 \mathrm{x} \mathrm{g}$, and then stored at $-80^{\circ} \mathrm{C}$. The concentrations of DAO, D-Lac, and Cry- 2 in the serum were analyzed using DAO, D-Lac and Cry-2 enzyme-linked immunosorbent assay (ELISA) assay kits (RD mouse DAO, D-Lac and $\alpha$-defensin-2 ELISA kits; R\&D Systems China Co., Ltd., Shanghai, China) according to the manufacturer's protocol.

Detection of intestinal concentrations of DAO, D-Lac, and Cry-2. The Ileal tissues were cut into smaller sections and grinded, and total proteins were extracted in phosphate-buffered saline (PBS) (containing $1 \mathrm{mM} \mathrm{KH}_{2} \mathrm{PO}_{4}, 155 \mathrm{mM} \mathrm{NaCl}$, $3 \mathrm{mM} \mathrm{Na} 2 \mathrm{HPO}_{4}-7 \mathrm{H}_{2} \mathrm{O}$ ), followed by centrifugation at $4{ }^{\circ} \mathrm{C}$ for $20 \mathrm{~min}$ at $13,400 \mathrm{x} \mathrm{g}$. The protein concentrations in the supernatant were quantified using a Micro BCA protein assay (Pierce; Thermo Fisher Scientific, Inc.). The concentrations of DAO, D-Lac and Cry-2 in the intestinal extracts were analyzed using ELISA assay kits according to the manufacturer's protocol.

Statistical analysis. Data are presented as the mean \pm standard deviation and were analyzed using SPSS 13.0 (SPSS, Inc., Chicago, IL, USA). One-way analysis of variance was used for the comparison of qualitative variables. The correlation between intestinal or serum levels of Cry-2 and intestinal injury scores, and the concentrations of intestinal and serum DAO and D-Lac, were calculated using Spearman's correlation analysis. $\mathrm{P}<0.05$ was considered to indicate a statistically significant difference.

\section{Results}

$2 D$ gel electrophoresis and protein identification. As described in our previous study (5), 2D gel electrophoresis was used to separate proteins extracted from the small intestines 


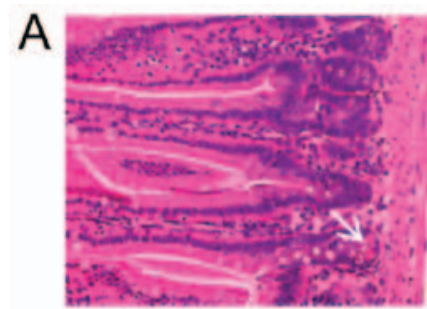

$37^{\circ} \mathrm{C}$

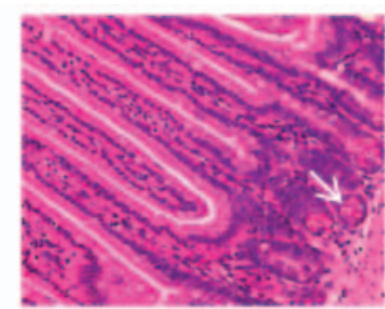

$40{ }^{\circ} \mathrm{C}$

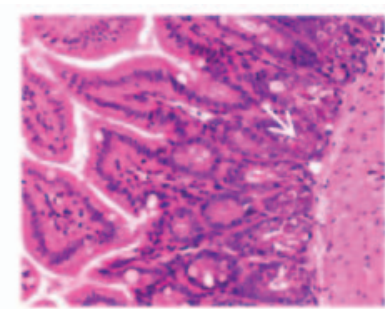

$42{ }^{\circ} \mathrm{C}$

B

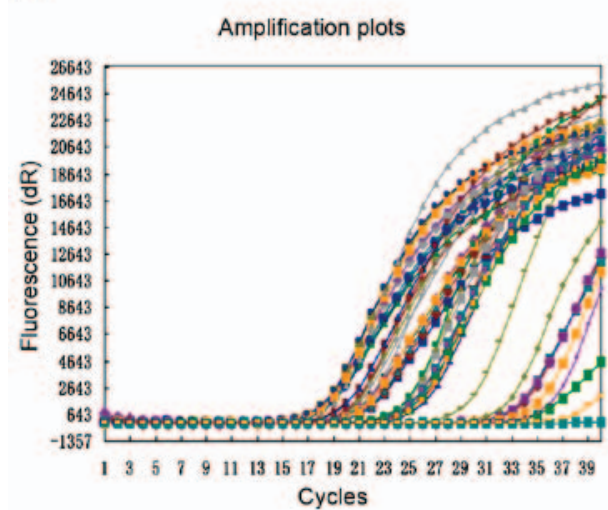

C

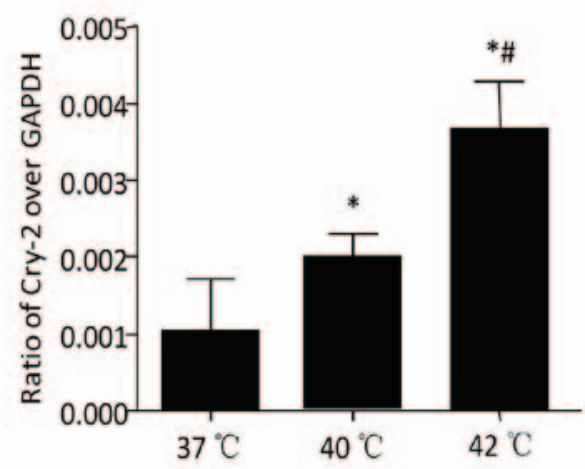

Figure 2. Histopathological changes in intestinal crypts and RT-qPCR identification of Cry-2 in mice subjected to heat stress. Male Balb/c mice were used to established heat-stressed mice in an incubator at $35.5 \pm 0.5^{\circ} \mathrm{C}$ and $60 \pm 5 \%$ humidity, which were then were divided into three groups: Sham-heated control mice $\left(37^{\circ} \mathrm{C}\right)$ and two groups of heat-stressed mice with core temperatures of 40 and $42^{\circ} \mathrm{C}$, respectively. (A) Representative images of hematoxylin and eosin-stained ileal crypts (magnification, $\mathrm{x} 400$ ). White arrows indicate defensin (red dye particles) secreted by paneth cells in small intestinal crypts. RT-qPCR analysis was used to examine mRNA levels of Cry-2. (B) RT-qPCR amplification curve. (C) Ratio of Cry-2 to GAPDH. Data are presented as the mean \pm standard deviation. One-way analysis of variance followed by the Newman-Keuls test was performed. ${ }^{*} \mathrm{P}<0.05$ vs. $37^{\circ} \mathrm{C}$ group; ${ }^{\prime \prime} \mathrm{P}<0.05$ vs. $40^{\circ} \mathrm{C}$ group $(\mathrm{n}=6$ ). Cry-2, cryptdin-2; RT-qPCR, reverse transcription-quantitative polymerase chain reaction.

of the control and heat-stressed mice. Among all protein spots analyzed using an ImageMaster 2D Elite (GE Healthcare Life Sciences) the $\mathrm{H} 1$ protein spots were found to be significantly altered between the controls and heat-stressed mice. The protein was successfully identified by MALDI-TOF MS and a subsequent comparative sequence search was performed in the Mascot database. An enlarged image of the H1 spot, subsequently identified as defensin-related Cry-2, on the 2D gel and its MALDI-TOF MS identification are shown in Fig. 1, respectively.

Histopathological changes in intestinal crypts and RT-qPCR identification of Cry-2 in heat-stressed mice. Male BALB/c mice were used to prepare the heat-stressed mice, which were divided into three groups: Sham-heated control mice $\left(37^{\circ} \mathrm{C}\right)$ and mice heated to a Tc of 40 or $42^{\circ} \mathrm{C}$, respectively. The ileal crypts were observed using a microscope following hematoxylin and eosin staining (magnification, $\mathrm{x} 400$ ). As shown in Fig. 2A, the level of defensin (red dye particles, indicated by white arrows) secreted by paneth cells in small intestinal crypts increased with HS. RT-qPCR analysis was used to examine the mRNA levels of Cry-2, the results of which were in accordance with the pathological changes induced by HS, the expression of Cry-2 was higher at a higher Tc (Fig. 2B). This finding was confirmed by the results of the $2 \mathrm{D}$-gel reported above.

$H S$-induced intestinal pathologic injury. The pathological changes in the intestines of mice in each group are shown in Fig. 3A. In the $37^{\circ} \mathrm{C}$ group and the $37^{\circ} \mathrm{C} / 6 \mathrm{~h}$ group, no significant damage was observed. Intestinal injury gradually increased with the increase in Tc, as shown by the aggravation of intestinal damage in the $40^{\circ} \mathrm{C}$ group and the $42^{\circ} \mathrm{C}$ group. In the $40^{\circ} \mathrm{C}$ group, marked epithelial necrosis was observed, with lesions exhibiting epithelial necrosis and villi desquamation. As the Tc increased to $42^{\circ} \mathrm{C}$, the damage to the villi increased. Intestinal damage was less extensive in the $40^{\circ} \mathrm{C} / 6 \mathrm{~h}$ group, compared with the damage in the $40^{\circ} \mathrm{C}$ group. A level of recovery among the villi was detected in the $40^{\circ} \mathrm{C} / 6 \mathrm{~h}$ group. By contrast, intestinal damage persisted following cooling in the heat-stressed mice whose $\mathrm{Tc}$ had reached $42^{\circ} \mathrm{C}$ during HS. Epithelial loss and villi desquamation were observed in the $42^{\circ} \mathrm{C} / 6 \mathrm{~h}$ group. Comparisons of intestinal injury scores showed similar results in terms of the morphological changes (Fig. 3B).

In addition to pathological changes, chemical markers of intestinal injury induced by HS were also detected. In this series, intestinal and serum concentrations of DAO were significantly increased in the $40^{\circ} \mathrm{C}$ group and the $42^{\circ} \mathrm{C}$ group, and increased further even following cooling treatment for $6 \mathrm{~h}$ (Fig. 3C and D), suggesting severe damage to the intestinal mucosa. In accordance with the changes in DAO, the levels of D-Lac increased in the serum and the intestines of the mice in the $40^{\circ} \mathrm{C}$ group and the $42^{\circ} \mathrm{C}$ group. Following cooling treatment, the concentrations of D-Lac also increased, compared with the concentrations in the $40^{\circ} \mathrm{C}$ group and the $42^{\circ} \mathrm{C}$ group (Fig. $3 \mathrm{E}$ and $\mathrm{F}$ ), which suggested that the permeability of the intestinal wall increased during HS and cooling treatment. 
A

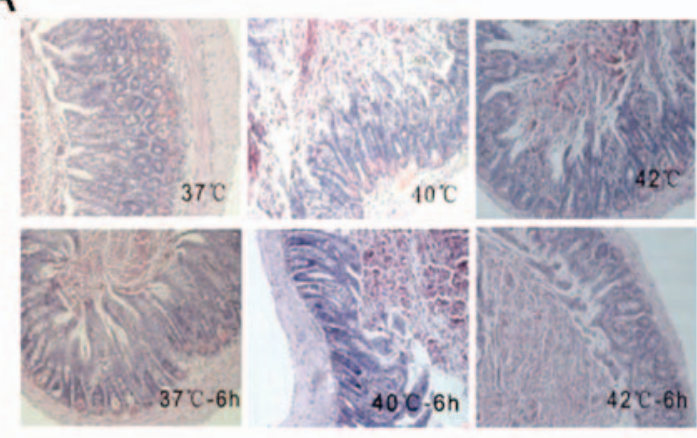

B

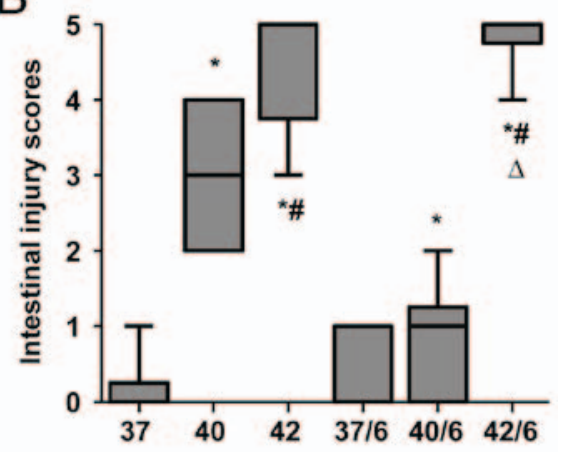

C

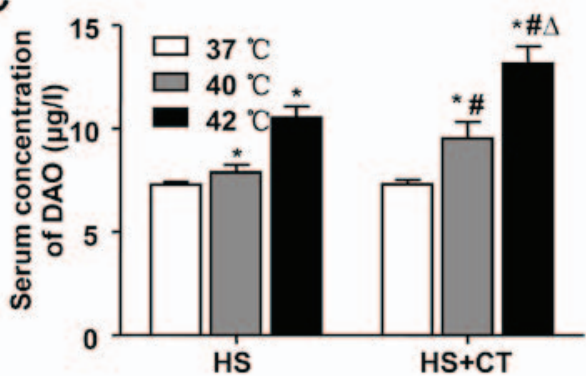

E

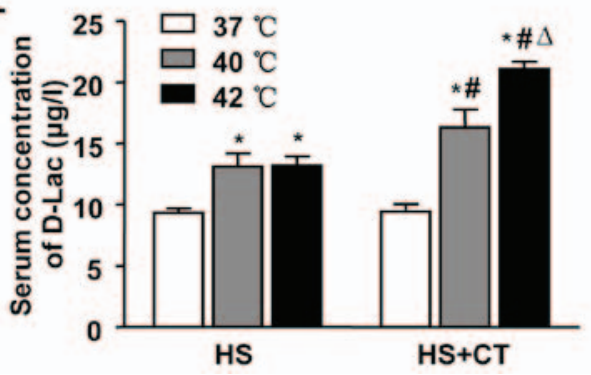

D

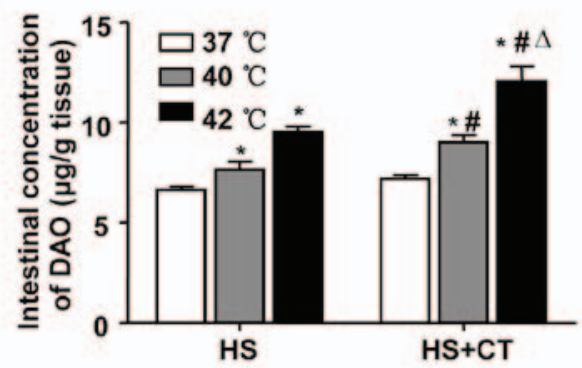

F

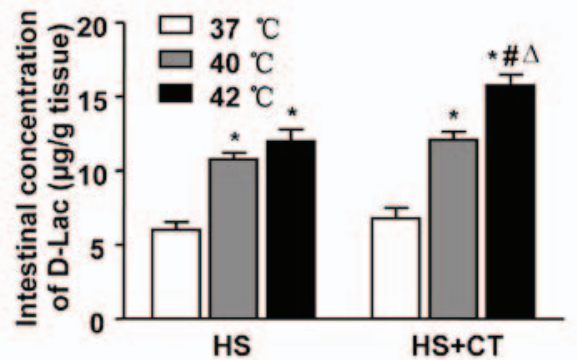

Figure 3. Effects of heat stress and cooling treatment on intestinal injury. Male Balb/c mice were used to establish heat-stressed mice, which were then divided into six groups: Sham-heated control mice $\left(37^{\circ} \mathrm{C}\right)$, mice heated with $\mathrm{Tc}$ at $40^{\circ} \mathrm{C}$ or $42^{\circ} \mathrm{C}$, and groups in which the animals were removed from the incubator and allowed to cool at an ambient temperature of $25 \pm 0.5^{\circ} \mathrm{C}$ for $6 \mathrm{~h}$ following core temperature reaching $40^{\circ} \mathrm{C}(40 / 6)$ or $42^{\circ} \mathrm{C}(42 / 6)$, or the sham control temperature (37/6). (A) Representative images of hematoxylin and eosin-stained ileal tissues (magnification, x200). (B) Morphological changes in mice ileal tissues were assessed and graded in a blinded-manner by two certified veterinary pathologists using the Chiu intestinal injury score. Concentrations of DAO in (C) serum and (D) ileal tissues, and those of D-LAC in the (E) serum and (F) ileal tissues were analyzed using an ELISA. Data are presented as the mean \pm standard deviation. One-way analysis of variance followed by the Newman-Keuls test was performed. ${ }^{*} \mathrm{P}<0.05$ vs. $37^{\circ} \mathrm{C}$ group; ${ }^{\prime} \mathrm{P}<0.05$ vs. $40^{\circ} \mathrm{C}$ group; ${ }^{\circ} \mathrm{P}<0.05$ vs. $42^{\circ} \mathrm{C}$ group $(n=6)$. DAO, diamine oxidase; D-Lac, D-lactic acid; HS, heat stress; CT, cooling treatment; ELISA, enzyme-linked immunosorbent assay.

HS increases concentrations of Cry-2 in serum and intestinal tissue in association with intestinal injury. Previous studies have shown that members of the defensin family, including Cry-2, are induced during several pathological conditions, including chronic colitis (28). As shown in Fig. 4, the results of the present study demonstrated that HS induced an increase in concentrations of Cry- 2 in the serum and intestines of the mice. Following cooling treatment, the levels of Cry-2 in the $40^{\circ} \mathrm{C} / 6 \mathrm{~h}$ group and the $42^{\circ} \mathrm{C} / 6 \mathrm{~h}$ group were significantly higher, compared with the concentrations in the $40^{\circ} \mathrm{C}$ group and $42^{\circ} \mathrm{C}$ group. These findings are consistent with the changes in intestinal injury, not only in terms of the pathological scores but also in terms of the chemical biomarkers.

To further confirm the correlation between intestinal or serum levels of Cry-2 and intestinal injury scores, the intestinal and serum concentrations of DAO and D-Lac were analyzed using Spearman's correlation analysis. As shown in Table I, the serum and intestinal levels of Cry-2 were positively correlated with the Chiu scores $(r=0.612 ; \mathrm{P}=0.0001 ; \mathrm{r}=0.541 ; \mathrm{P}=0.0006$, respectively). The findings also suggested that a significantly positive correlation existed between the levels of Cry-2 and D-Lac ( $r=0.778$ in serum; $r=0.850$ in intestines). Similarly, correlations were observed between the levels of Cry-2 and DAO in the serum and the intestines $(\mathrm{r}=0.567 ; \mathrm{P}=0097$; $\mathrm{r}=0.528 ; \mathrm{P}=0.0074$, respectively). These results indicated that Cry-2 may be a biomarker of HS-induced intestinal injury.

Ulinastatin protects against HS-induced intestinal damage. To determine the possible protective role of ulinastatin on intestinal injury in the heat-stressed mice, $100,000 \mathrm{U} / \mathrm{kg}$ ulinastatin was administered by intraperitoneal injection as soon as the Tc reached $42^{\circ} \mathrm{C}$. Cooling treatments for $6 \mathrm{~h}$ also were performed, and intestinal pathologic changes, Chiu scores, and serum and intestinal biomarkers (DAO and 
Table I. Correlation analysis between Cry-2 and intestinal injury.

Spearman's rank

\begin{tabular}{|c|c|c|c|c|}
\hline Cry-2 expression & Variable & $\mathrm{n}$ & Correlation coefficient & Significance (two-tailed) \\
\hline \multirow[t]{3}{*}{ Serum } & Chiu score & 36 & $0.612^{\mathrm{a}}$ & 0.0001 \\
\hline & $\mathrm{DAO}$ & 36 & $0.567^{\mathrm{a}}$ & 0.0097 \\
\hline & D-Lac & 36 & $0.778^{\mathrm{a}}$ & $<0.0001$ \\
\hline \multirow[t]{3}{*}{ Intestine } & Chiu score & 36 & $0.541^{\mathrm{a}}$ & 0.0006 \\
\hline & $\mathrm{DAO}$ & 36 & $0.528^{\mathrm{a}}$ & 0.0074 \\
\hline & D-Lac & 36 & $0.850^{\mathrm{a}}$ & $<0.0001$ \\
\hline
\end{tabular}

${ }^{\mathrm{a}} \mathrm{P}<0.01$ (two-tailed) was considered statistically significant. Cry-2, cryptdin-2; DAO, diamine oxidase; D-Lac, D-lactic acid.
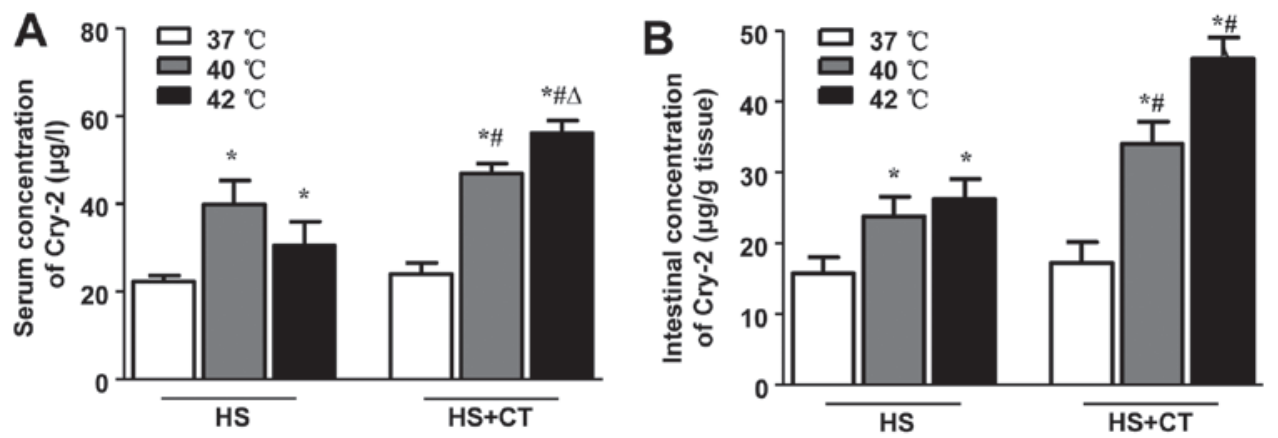

Figure 4. Changes in concentrations of Cry-2 in the (A) serum and (B) intestines of mice exposed to heat stress followed by cooling treatment. Male Balb/c mice were used to establish heat-stressed mice, which were then divided into six groups: Sham-heated control mice $\left(37^{\circ} \mathrm{C}\right)$, mice heated with Tc at $40^{\circ} \mathrm{C}$ or $42^{\circ} \mathrm{C}$, and groups in which the animals were removed from the incubator and allowed to cool at an ambient temperature of $25 \pm 0.5^{\circ} \mathrm{C}$ for $6 \mathrm{~h}$ following core temperature reaching $40^{\circ} \mathrm{C}(40 / 6)$ or $42^{\circ} \mathrm{C}(42 / 6)$, or the sham control temperature (37/6). Concentrations of Cry-2 in serum and ileal tissues were analyzed using ELISA. Data are presented as the mean \pm standard deviation. One-way analysis of variance followed by the Newman-Keuls test was performed. ${ }^{*} \mathrm{P}<0.05$ vs. $37^{\circ} \mathrm{C}$ group; ${ }^{\#} \mathrm{P}<0.05$ vs. $40^{\circ} \mathrm{C}$ group; ${ }^{\wedge} \mathrm{P}<0.05$ vs. $42^{\circ} \mathrm{C}$ group $(\mathrm{n}=6)$. Cry-2, cryptdin- 2 ; HS, heat stress; CT, cooling treatment; ELISA, enzyme-linked immunosorbent assay.

D-lac) were determined (Fig. 5). Compared with the $42^{\circ} \mathrm{C} / 6 \mathrm{~h}$ group, pathological sections from the $42^{\circ} \mathrm{C} / 6 \mathrm{~h}+\mathrm{UTI}$ group showed that villi damage was mitigated by ulinastatin treatment. This was confirmed by changes in the villi length. Villi swelling and vascular congestion were also moderated in the $42^{\circ} \mathrm{C} / 6 \mathrm{~h}+\mathrm{UTI}$ group. Similar changes were observed in the serum and intestinal levels of DAO and D-Lac. These results demonstrated that ulinastatin treatment may be beneficial in mitigating HS-induced intestinal damage.

Ulinastatin decreases serum and intestinal concentrations of Cry-2 in correlation with intestinal injury. To investigate the effects of ulinastatin on Cry-2, the present study measured serum and intestinal levels of Cry-2 following the treatments involving ulinastatin and cooling. The results showed that ulinastatin decreased the HS-induced serum and intestinal concentrations of Cry-2 (Fig. 6). Spearman's correlation analysis was also performed, and, as shown in Table II, the serum and intestinal levels Cry-2 were positively correlated with Chiu scores $(\mathrm{r}=0.728 ; \mathrm{P}<0.001 ; \mathrm{r}=0.651 ; \mathrm{P}=0.001$, respectively). The results also suggested significant positive correlations between the levels of Cry-2 and D-Lac ( $r=0.838$ in serum; $r=0.877$ in intestine). Similar correlations were also observed between the levels of Cry- 2 and DAO in the serum and the intestines $(\mathrm{r}=0.694 ; \mathrm{P}=001 ; \mathrm{r}=0.780 ; \mathrm{P}<0.001$, respectively). These results further established the correlation between the levels of Cry-2 and the severity of intestinal damage, even following ulinastatin treatment. In addition, the results provided further confirmation that Cry- 2 may be a novel biomarker of HS-induced intestinal injury.

\section{Discussion}

In our previous study, 2D gel electrophoresis was used to identify various $\mathrm{HS}$-induced intestinal proteins. The present study confirmed for the first time, to the best of our knowledge, that HS upregulated the expression of Cry-2. The concentrations of Cry-2 in the serum and intestines were positively correlated with the severity of HS-induced intestinal damage. In addition, ulinastatin protected the intestines from HS-induced injury and downregulated the expression of Cry-2. This reduced expression of Cry-2 was also correlated with changes in the degree of intestinal injury. Therefore, Cry-2 may be involved in and may be a novel predictor of HS-induced intestinal injury.

Severe HS, including that in heatstroke, has been reported to have a direct cytotoxic effect and to lead to organ damage. HS also causes gastrointestinal dysfunction, which is important in the pathophysiological process of heatstroke (4). The results of the present study demonstrated that HS can lead to intestinal damage, as shown by the pathological changes and 
A

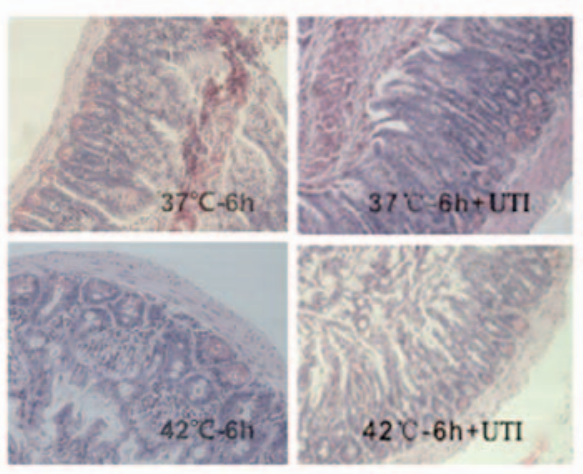

C

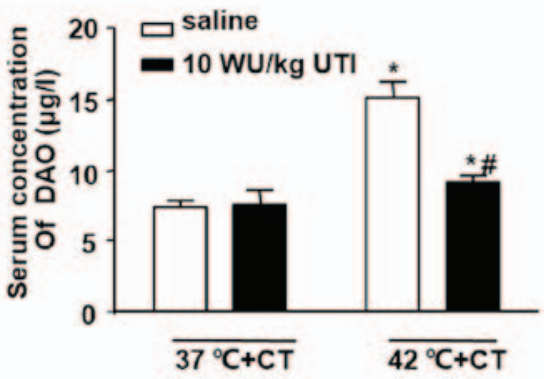

E

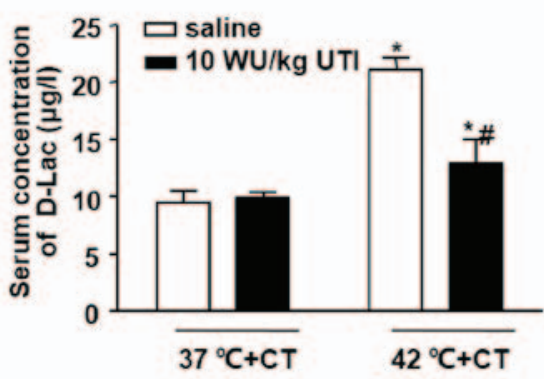

B

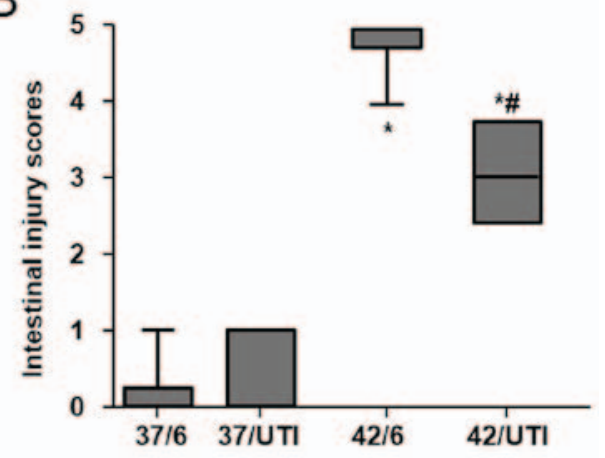

D

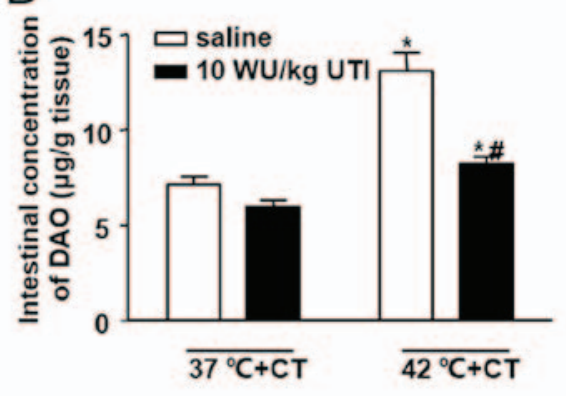

$\mathrm{F}$

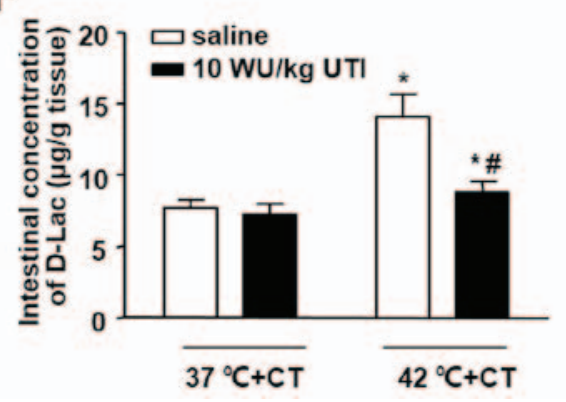

Figure 5. Effects of UTI on intestinal injury and concentrations of DAO and D-Lac in mice subjected to heat stress and cooling treatment. Heat-stressed mice were prepared and administered with cooling treatment for $6 \mathrm{~h}$ following core temperature reaching 42 ${ }^{\circ} \mathrm{C}(42 / 6)$. Sham-heated control mice were placed at an ambient temperature for the same time (37/6). UTI was administered by intraperitoneal injection at a dose of $100,000 \mathrm{U} / \mathrm{kg}$ when core temperature reached $42^{\circ} \mathrm{C}$ $(42+\mathrm{UTI})$ or used at same time in sham-heated control mice (37+UTI). Identical volumes of saline were used in the 37/6 and 42/6 groups. (A) Representative images of hematoxylin and eosin-stained ileal tissues (magnification, x200). (B) Morphological changes in mice ileal tissues were assessed and graded in a blinded-manner by two certified veterinary pathologists using the Chiu intestinal injury score. DAO concentrations in (C) serum and (D) ileal tissues, and D-Lac concentrations in (E) serum and (F) ilieal tissues were analyzed using ELISA. Data are presented as the mean \pm standard deviation. One-way analysis of variance followed by the Newman-Keuls test was performed. $\mathrm{P}<0.05$ vs. 37/6 group; ${ }^{*} \mathrm{P}<0.05$ vs. $42 / 6$ group (n=6). UTI, ulinastatin; $\mathrm{DAO}$, diamine oxidase; D-Lac, D-lactic acid; HS, heat stress; CT, cooling treatment; ELISA, enzyme-linked immunosorbent assay.

A

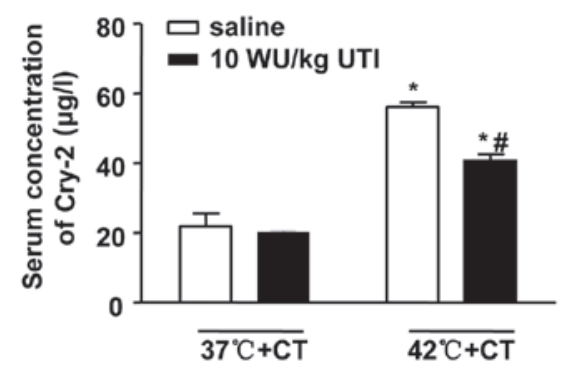

B

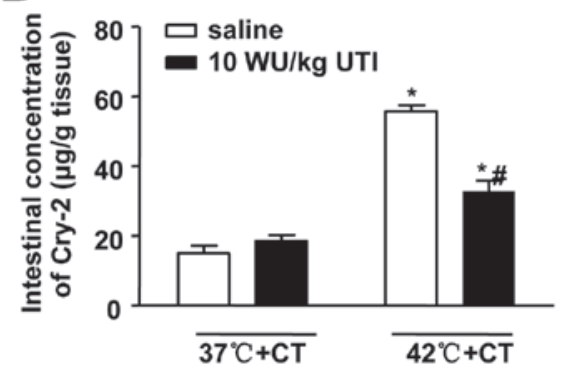

Figure 6. Effects of UTI on intestinal and serum concentrations of Cry-2 in mice subjected to heat stress and cooling treatment. Heat-stressed mice were prepared and underwent cooling treatment for $6 \mathrm{~h}$ following core temperature reached $42^{\circ} \mathrm{C}$. Sham-heated control mice were placed at an ambient temperature for the same time. UTI was administered by intraperitoneal injection at a dose of $100,000 \mathrm{U} / \mathrm{kg}$ when core temperature reached $42^{\circ} \mathrm{C}$ or at same time in sham-heated control mice. Identical volumes of saline were used as the vehicle for UTI administration. Concentrations of Cry-2 in (A) serum and (B) ileal tissues were analyzed using ELISA. Data are presented as the mean \pm standard deviation. One-way analysis of variance followed by the Newman-Keuls test was performed. ${ }^{\mathrm{P}}<0.05$ vs. $37^{\circ} \mathrm{C}+$ saline group; ${ }^{\prime} \mathrm{P}<0.05$ vs. $42^{\circ} \mathrm{C}+$ saline group $(\mathrm{n}=6)$. Cry-2, cryptdin-2; UTI, ulinastatin; HS, heat stress; CT, cooling treatment; ELISA, enzyme-linked immunosorbent assay. 
Table II. Correlation analysis between Cry-2 and intestinal injury following ulinastatin treatment.

Spearman's rank

\begin{tabular}{llccr}
\cline { 3 - 4 } Cry-2 expression & Variable & $\mathrm{n}$ & Correlation coefficient & Significance (two-tailed) \\
\hline Serum & Chiu score & 24 & $0.728^{\mathrm{a}}$ & $<0.001$ \\
& DAO & 24 & $0.694^{\mathrm{a}}$ & 0.001 \\
& D-Lac & 24 & $0.838^{\mathrm{a}}$ & $<0.001$ \\
Intestine & Chiu score & 24 & $0.651^{\mathrm{a}}$ & 0.001 \\
& DAO & 24 & $0.780^{\mathrm{a}}$ & $<0.001$ \\
& D-Lac & 24 & $0.877^{\mathrm{a}}$ & $<0.001$ \\
\hline
\end{tabular}

${ }^{\text {a }}<<0.01$ (two-tailed) was considered statistically significant. Cry-2, cryptdin-2; DAO, diamine oxidase; D-Lac, D-lactic acid.

the increased concentrations of DAO and D-Lac. Physically, the intestinal barrier can prevent colonization by bacteria entering the systemic circulation. The dysfunction of this barrier is important in promoting heat-induced MODS, as is the pathophysiologic process in sepsis (29). The basal levels of DAO and D-Lac are usually low in the systemic circulation, and are usually observed only in the intestines. In the present study, the increased concentrations of DAO and D-Lac suggested that the permeability of the intestinal wall was increased following HS. Pathological sections in the present study revealed decreased length of villi, with the presence of denuded villi in severe cases. Damage to the intestinal mucosa allows endotoxins to escape the intestinal lumen, leading to secondary bacteremia.

The mechanism of HS-induced intestinal damage remains to be fully elucidated. Current views focus mainly on direct thermal stresses (30), followed by insults from inflammatory and ischemia-induced reactive oxygen species.

In vitro experiments have shown that direct $\mathrm{HS}$ can induce intestinal cell apoptosis (30) and inflammation via disturbance of the B-cell lymphoma-2 (Bcl-2)-associated X protein/Bcl-2 balance and activation of the nuclear factor- $\kappa \mathrm{B}(\mathrm{NF}-\kappa \mathrm{B})$ signaling pathway (31). HS can downregulate tight junction proteins, resulting in increased intestinal permeability. The heat stroke-induced systemic inflammatory cascade is also important in the pathology. Our previous study demonstrated that levels of pro-inflammatory cytokines were increased in the intestines and positively correlated with intestinal injury scores (25). Enterocytes are sensitive to oxygen restriction, and heat-induced splanchnic vessel contraction leads to the production of reactive oxygen species and stress in the endoplasmic reticulum $(32,33)$ particularly at the tips of the villi. This ischemic insult aggravates intestinal damage and dysfunction of the intestinal barrier. As reported in previous studies $(25,34)$, the results of the present study showed that intestinal damage was aggravated following cooling treatment. This may result from gut reperfusion during the cooling treatment and the sustained systemic inflammatory cascade $(35,36)$. The inflammatory responses are not only stimulated by thermal stress but also are activated by gut endotoxins released due to dysfunction of the intestinal barrier.

Defensin-related Cry-2, an intestinal $\alpha$-defensin, was previously found to be upregulated in the small intestines of mice with heatstroke (5). The enteric surface barrier is crucial in preventing the translocation of macromolecules and bacteria from the colonized mouse gut lumen. In the gut lumen, paneth cells are key members and the main producers of antimicrobial peptides, primarily $\alpha$-defensins, which are termed cryptdins in mice (37). Cryptdins, which have potent microbiacidal activity, are secreted into the lumen in response to bacterial stimuli (38). The results of the present study confirmed this change by histopathological detection and RT-qPCR analysis. In addition, HS increased the serum and intestinal concentrations of Cry-2, and was correlated with changes in Chiu scores, and the serum and intestinal concentrations of D-Lac and DAO. Therefore, the concentration of Cry-2 may indicate intestinal damage, and the level of Cry-2 may vary according to the severity of injury.

The results of the present study confirmed that the concentration of Cry- 2 was increased in heat-stressed mice and that the concentrations of cryptdin were positively correlated with the extent of lesions. However, whether increased cryptdin results from pathological factors or whether cryptdin induces injury remains to be elucidated. It appears that both are involved. A certain concentration of cryptdin is necessary to induce antimicrobial activity. During infection and inflammation, the translocation of multitude microbes from the enteric cavity to the circulation is increased. According to the Wehkamp et al raised 'on-demand' mechanism (39), the increased defensin may be protective to the host. Large numbers of copies of cryptdin genes are present in paneth cells, even under resting conditions (40), which may explain the rapid response of cryptdins to infection.

Cryptdins mediate enteric innate immunity, and their absence may contribute to enhanced susceptibility to infection $(7,40)$. Cry-2 can combat infections and augment the activity of conventional antibiotics in vitro and in vivo $(8,9)$. $\alpha$-defensins may suppress inflammation by controlling the production of interleukin-1 $\beta$ (IL-1 $\beta$ ) (11). By contrast, defensin is cytotoxic and pro-inflammatory. This cytotoxicity is based on the interaction of positively charged peptides with negatively charged phospholipid bilayers of cell membranes, leading to the disorganization of the cytomembrane (41). Under resting conditions, defensins have no effect on normal cells, and it is unclear whether alteration of the defensin recognition site or cytomembrane potential occurs during 
the pathologic process of heatstroke. In addition, defensin has effects on the regulation of inflammation, including the upregulation of IL-8 and inducing the chemotactic activity of T lymphocytes $(12,41)$. Ulinastatin has multiple functions, including reducing inflammation, regulating immune responses and alleviating ischemia-reperfusion injury; it has been used to treat pancreatitis, sepsis, shock, and cardiac and renal ischemic reperfusion (41-43). The present study showed that ulinastatin effectively alleviated heat-induced intestinal damage, even during cooling treatment, resulting in decreased pathological injury and reduced concentrations of DAO and D-Lac. A previous study showed that ulinastatin pretreatment reduced the inflammatory exudation in heat-induced acute lung injury (24). Ulinastatin's anti-inflammatory effects include inactivating the elastase secreted by neutrophils, decreasing inflammatory mediators and downregulating inflammatory transcription factors, including $\mathrm{NF}-\kappa \mathrm{B}(19,22,45)$. Under inflammatory conditions, ulinastatin can attenuate dysfunctions of the endothelial barrier by upregulating the expression of vascular endothelial-cadherin; it also prevents endothelial apoptosis $(21,46,47)$. These effects against hyperpermeability may account for the protective effect of ulinastatin against heat-induced damage.

Ulinastatin may also protect the intestines from ischemia induced by splanchnic vessel contraction following heat stimuli. Kato et al found that ulinastatin has lysosomal membrane-stabilizing properties (48), which can prevent the rupture of lysosomes under pathological conditions. In the reperfusion state, ulinastatin maintains energy production by reducing the severity of mitochondrial dysfunction (44). The present study confirmed that post-treatment ulinastatin had a protective effect in heat-stressed mice, and the protection may result from its anti-inflammatory and anti-ischemic functions. These findings indicated that ulinastatin may be used in the treatment of patients with heatstroke.

Defensins are induced via cell receptors, which recognize pathogen-associated molecular patterns, including Toll-like receptors (TLRs), TLR2 and TLR4 (49). The results of the present study showed that ulinastatin decreased the level of cryptdin, and this effect may be associated with the suppressive effect of on the activity of TLR4 (50). Nucleotide-binding oligomerization domain protein 2 is another crucial sensor protein involved in the production of defensin via the NF- $\mathrm{B}$ pathway $(49,51)$. Therefore, ulinastatin may downregulate the expression of Cry-2 by inhibiting NF- $\mathrm{NB}$ (22). Ulinastatin exerts a protective effect on cells via inhibiting the release of cytochrome $\mathrm{c}$ and activation of caspase-3, which are beneficial to vascular permeability (46). The decreased concentration of Cry- 2 may be the result of reduced permeability following ulinastatin treatment.

In conclusion, the present study confirmed for the first time, to the best of our knowledge, that HS in a mouse model upregulated the expression of Cry-2, and that the serum and intestinal concentrations of Cry- 2 were positively correlated with the severity of HS-induced intestinal damage. It was also found that ulinastatin protected the intestines from HS-induced injury and concurrently downregulated the expression of Cry-2. The latter was also correlated with changes in intestinal injury. Therefore, ulinastatin administration may be beneficial for patients with heatstroke, and Cry-2 may be a novel predictor of HS-induced intestinal injury.

\section{Acknowledgements}

The present study was supported by grants from the National Natural Science Foundation of China (grant nos. 81571940 and 81101467), the Project Team of Natural Science Foundation of Guangdong Province (grant no. s2013030013217), the Project of Medical Research of PLA (grant no. BWS12J108), the Guangzhou Science and Technology Planning Project of China (grant no.201504281714528) and the Natural ScienceFoundation of Guangdong Province (grant no. 10151001002000001).

\section{References}

1. Leon LR and Bouchama A: Heat stroke. Compr Physiol 5: 611-647, 2015.

2. Sucholeiki R: Heatstroke. Semin Neurol 25: 307-314, 2005.

3. Lambert GP, Gisolfi CV, Berg DJ, Moseley PL, Oberley LW and Kregel KC: Selected contribution: Hyperthermia-induced intestinal permeability and the role of oxidative and nitrosative stress. J Appl Physiol (1985) 92: 1750-1761; discussion 1749, 2002.

4. Yang P-C, He S-H and Zheng P-Y: Investigation into the signal transduction pathway via which heat stress impairs intestinal epithelial barrier function. J Gastroenterol Hepatol 22: 1823-1831, 2007.

5. Liu Z, Liu JH, Liu Y, Tang Y, Meng F, Sun X, Tang J, Wang JH and $\mathrm{Su} \mathrm{L}$ : Proteomic analysis and identification of intestinal FBP as a predictor of gut dysfunction during heatstroke in mice. J Surg Res 173: 332-340, 2012.

6. Ouellette AJ, Hsieh MM, Nosek MT, Cano-Gauci DF, Huttner KM, Buick RN and Selsted ME: Mouse Paneth cell defensins: Primary structures and antibacterial activities of numerous cryptdin isoforms. Infect Immun 62: 5040-5047, 1994

7. Ouellette AJ, Satchell DP, Hsieh MM, Hagen SJ and Selsted ME Characterization of luminal paneth cell alpha-defensins in mouse small intestine. Attenuated antimicrobial activities of peptides with truncated amino termini. J Biol Chem 275: 33969-33973, 2000.

8. Preet S, Verma I and Rishi P: Cryptdin-2: A novel therapeutic agent for experimental Salmonella typhimurium infection. J Antimicrob Chemother 65: 991-994, 2010.

9. Singh AP, Prabha V and Rishi P: Efficacy of cryptdin-2 as an adjunct to antibiotics from various generations against Salmonella. Indian J Microbiol 54: 323-328, 2014.

10. Inoue R, Tsuruta T, Nojima I, Nakayama K, Tsukahara T and Yajima T: Postnatal changes in the expression of genes for cryptdins 1-6 and the role of luminal bacteria in cryptdin gene expression in mouse small intestine. FEMS Immunol Med Microbiol 52: 407-416, 2008.

11. Shi J, Aono S, Lu W, Ouellette AJ, Hu X, Ji Y, Wang L, Lenz S, van Ginkel FW, Liles M, et al: A novel role for defensins in intestinal homeostasis: Regulation of IL-1beta secretion. J Immunol 179: 1245-1253, 2007.

12. Lee HT, Kim M, Kim JY, Brown KM, Ham A, D'Agati VD and Mori-Akiyama Y: Critical role of interleukin-17A in murine intestinal ischemia-reperfusion injury. Am J Physiol Gastrointest Liver Physiol 304: G12-G25, 2013.

13. Miyoshi J, Miyamoto H, Goji T, Taniguchi T, Tomonari T, Sogabe M, Kimura T, Kitamura S, Okamoto K, Fujino Y, et al: Serum diamine oxidase activity as a predictor of gastrointestinal toxicity and malnutrition due to anticancer drugs. J Gastroenterol Hepatol 30: 1582-1590, 2015.

14. Tan S, Yokoyama Y, Dickens E, Cash TG, Freeman BA and Parks DA: Xanthine oxidase activity in the circulation of rats following hemorrhagic shock. Free Radic Biol Med 15: 407-414, 1993.

15. Demircan M, Cetin S, Uguralp S, Sezgin N, Karaman A and Gozukara EM: Plasma D-lactic acid level: A useful marker to distinguish perforated from acute simple appendicitis. Asian J Surg 27: 303-305, 2004.

16. Smith SM, Eng RH and Buccini F: Use of D-lactic acid measurements in the diagnosis of bacterial infections. J Infect Dis 154: 658-664, 1986.

17. Chen X, Wang Y, Luo H, Luo Z, Liu L, Xu W, Zhang T, Yang N, Long $\mathrm{X}, \mathrm{Zhu} \mathrm{N}$, et al: Ulinastatin reduces urinary sepsis related inflammation by upregulating IL-10 and downregulating TNF- $\alpha$ levels. Mol Med Rep 8: 29-34, 2013. 
18. Wang SY, Li ZJ, Wang X, Li WF and Lin ZF: Effect of ulinastatin on HMGB1 expression in rats with acute lung injury induced by sepsis. Genet Mol Res 14: 4344-4353, 2015.

19. Nakatani K, Takeshita S, Tsujimoto H, Kawamura Y and Sekine I: Inhibitory effect of serine protease inhibitors on neutrophilmediated endothelial cell injury. J Leukoc Biol 69: 241-247, 2001

20. Xu CE, Zhang MY, Zou CW and Guo L: Evaluation of the pharmacological function of ulinastatin in experimental animals. Molecules 17: 9070-9080, 2012.

21. Li C, Ma D, Chen M, Zhang L, Zhang L, Zhang J, Qu X and Wang C: Ulinastatin attenuates LPS-induced human endothelial cells oxidative damage through suppressing JNK/c-Jun signaling pathway. Biochem Biophys Res Commun 474: 572-578, 2016.

22. Feng C, Li B, Wang LL, Chen LI, Zhou X, Lv FQ and Li TS: Effect of peritoneal lavage with ulinastatin on the expression of $\mathrm{NF}-\kappa \mathrm{B}$ and TNF- $\alpha$ in multiple organs of rats with severe acute pancreatitis. Exp Ther Med 10: 2029-2034, 2015.

23. Atal SS and Atal S: Ulinastatin - a newer potential therapeutic option for multiple organ dysfunction syndrome. J Basic Clin Physiol Pharmacol 27: 91-99, 2016.

24. Zhou G, Xu Q, Liu Y, Wang Z, Su L and Guo X: Protective effects of ulinastatin against acute lung injury induced by heatstroke in mice. Nan Fang Yi Ke Da Xue Xue Bao 35: 1277-1282, 2015 (In Chinese).

25. Liu Z, Sun X, Tang J, Tang Y, Tong H, Wen Q, Liu Y and Su L: Intestinal inflammation and tissue injury in response to heat stress and cooling treatment in mice. Mol Med Rep 4: 437-443, 2011.

26. Chiu CJ, McArdle AH, Brown R, Scott HJ and Gurd FN: Intestinal mucosal lesion in low-flow states. I. A morphological, hemodynamic, and metabolic reappraisal. Arch Surg 101: 478-483, 1970.

27. Livak KJ and Schmittgen TD: Analysis of relative gene expression data using real-time quantitative PCR and the 2(-Delta Delta C(T)) method. Methods 25: 402-408, 2001.

28. Rahman A, Fahlgren A, Sundstedt C, Hammarström S, Danielsson A and Hammarström ML: Chronic colitis induces expression of $\beta$-defensins in murine intestinal epithelial cells. Clin Exp Immunol 163: 123-130, 2011.

29. Gathiram P, Wells MT, Raidoo D, Brock-Utne JG and Gaffin SL: Portal and systemic plasma lipopolysaccharide concentrations in heat-stressed primates. Circ Shock 25: 223-230, 1988.

30. Tang J, Jiang Y, Tang Y, Chen B, Sun X, Su L and Liu Z: Effects of propofol on damage of rat intestinal epithelial cells induced by heat stress and lipopolysaccharides. Braz J Med Biol Res 46: 507-512, 2013

31. Liu Y, Zhou G, Wang Z, Guo X, Xu Q, Huang Q and Su L: NF-кB signaling is essential for resistance to heat stress-induced early stage apoptosis in human umbilical vein endothelial cells. Sci Rep 5: 13547, 2015.

32. Hall DM, Baumgardner KR, Oberley TD and Gisolfi CV: Splanchnic tissues undergo hypoxic stress during whole body hyperthermia. Am J Physiol 276: G1195-G1203, 1999.

33. Yin P, Xu J, He S, Liu F, Yin J, Wan C, Mei C, Yin Y, Xu X and $\mathrm{Xia} Z$ : Endoplasmic reticulum stress in heat- and shake-induced injury in the rat small intestine. PLoS One 10: e0143922, 2015.

34. Johnson JS, Sapkota A and Lay DC Jr: Rapid cooling after acute hyperthermia alters intestinal morphology and increases the systemic inflammatory response in pigs. J Appl Physiol (1985) 120 $1249-1259,2016$

35. Starkie RL, Hargreaves M, Rolland J and Febbraio MA: Heat stress, cytokines, and the immune response to exercise. Brain Behav Immun 19: 404-412, 2005.

36. Welc SS, Judge AR and Clanton TL: Skeletal muscle interleukin-6 regulation in hyperthermia. Am J Physiol Cell Physiol 305: C406-C413, 2013.
37. Dupont A, Heinbockel L, Brandenburg K and Hornef MW: Antimicrobial peptides and the enteric mucus layer act in concert to protect the intestinal mucosa. Gut Microbes 5: 761-765, 2014.

38. Ayabe T, Satchell DP, Wilson CL, Parks WC, Selsted ME and Ouellette AJ: Secretion of microbicidal alpha-defensins by intestinal Paneth cells in response to bacteria. Nat Immunol 1: 113-118, 2000.

39. Wehkamp J, Schmid M and Stange EF: Defensins and other antimicrobial peptides in inflammatory bowel disease. Curr Opin Gastroenterol 23: 370-378, 2007.

40. Mastroianni JR and Ouellette AJ: Alpha-defensins in enteric innate immunity: Functional Paneth cell alpha-defensins in mouse colonic lumen. J Biol Chem 284: 27848-27856, 2009.

41. Mattar EH, Almehdar HA, Yacoub HA, Uversky VN and Redwan EM: Antimicrobial potentials and structural disorder of human and animal defensins. Cytokine Growth Factor Rev 28: 95-111, 2016.

42. Zhang C, Wang Y, Fu W, Zhang W, Wang T and Qin H: A metaanalysis on the effect of ulinastatin on serum levels of C-reactive protein, interleukin 6, and tumor necrosis factor alpha in Asian patients with acute pancreatitis. Genet Test Mol Biomarkers 20: 118-124, 2016.

43. Huang Y, Xie K, Zhang J, Dang Y and Qiong Z: Prospective clinical and experimental studies on the cardioprotective effect of ulinastatin following severe burns. Burns 34: 674-680, 2008.

44. Masuda T, Sato K, Noda C, Ikeda KM, Matsunaga A, Ogura MN, Shimizu K, Nagasawa H, Matsuyama N and Izumi T: Protective effect of urinary trypsin inhibitor on myocardial mitochondria during hemorrhagic shock and reperfusion. Crit Care Med 31: 1987-1992, 2003

45. Wang LZ, Luo MY, Zhang JS, Ge FG, Chen JL and Zheng CQ: Effect of ulinastatin on serum inflammatory factors in Asian patients with acute pancreatitis before and after treatment: A meta-analysis . Int J Clin Pharmacol Ther 54: 890-898, 2016.

46. Wang L, Huang X, Kong G, Xu H, Li J, Hao D, Wang T, Han S, Han C, Sun Y, et al: Ulinastatin attenuates pulmonary endothelial glycocalyx damage and inhibits endothelial heparanase activity in LPS-induced ARDS. Biochem Biophys Res Commun 478: 669-675, 2016.

47. Lin B, Liu Y, Li T, Zeng K, Cai S, Zeng Z, Lin C, Chen Z and Gao Y: Ulinastatin mediates protection against vascular hyperpermeability following hemorrhagic shock. Int J Clin Exp Pathol 8: 7685-7693, 2015.

48. Kato Y, Kudo M, Shinkawa T, Mochizuki H, Isaji M, Shiromizu I and Hoshida K: Role of O-linked carbohydrate of human urinary trypsin inhibitor on its lysosomal membrane-stabilizing property. Biochem Biophys Res Commun 243: 377-383, 1998.

49. Lehrer RI: Multispecific myeloid defensins. Curr Opin Hematol 14: 16-21, 2007.

50. Gao C, Li R and Wang S: Ulinastatin protects pulmonary tissues from lipopolysaccharide-induced injury as an immunomodulator. J Trauma Acute Care Surg 72: 169-176, 2012.

51. Kobayashi KS, Chamaillard M, Ogura Y,Henegariu O, Inohara N, Nuñez G and Flavell RA: Nod2-dependent regulation of innate and adaptive immunity in the intestinal tract. Science 307: 731-734, 2005.

This work is licensed under a Creative Commons Attribution-NonCommercial-NoDerivatives 4.0 International (CC BY-NC-ND 4.0) License. 